\title{
The Role of the Mammillary Bodies in Memory: A Case of Amnesia Following Bilateral Resection
}

\author{
Leigh J. Beglinger* \\ Marc W. Haut** \\ Michael W. Parsons ${ }^{* *}$ \\ * Department of Psychiatry, University of \\ lowa Carver College of Medicine \\ ** Department of Behavioral Medicine and \\ Psychiatry, Department of Neurology, and \\ Department of Radiology, West Virginia \\ University School of Medicine, Morgantown, \\ West Virginia
}

USA

\begin{abstract}
Background and Objectives: Craniopharyngioma (CP) patients typically show good neuropsychiatric outcome following tumor resection. We present the case of a 51-year old woman who sustained damage to white matter pathways during surgery resulting in a disconnection of the Papez circuit (loss of bilateral mammillary bodies, columns of the fornix and mammillothalamic tracts).

Methods and Results: Neuropsychological evaluations were completed at 10 and 30 weeks post-operatively, and indicated both retrograde and severe anterograde amnesia, as well as persistent depression. At the second evaluation, most cognitive deficits had improved, but memory and mood deficits remained. Metamemory and priming remained intact.

Conclusions: This case illustrates a profound neuropsychiatric morbidity associated with a surgery that is typically considered benign and confirms the well-known dissociation between explicit recollection of newly learned information and less conscious forms of learning and memory. This rare pathology provides further information regarding the role of the mammillary bodies in memory.
\end{abstract}

Received 10 August 2005

Accepted 3 February 2006

Despite being a histologically benign tumor, the adhesive nature and suprasellar location of craniopharyngiomas (CP), with proximity to subcortical structures and critical memory pathways, presents cognitive and psychiatric risks for patients undergoing surgical resection. As CPs predominate in the first two decades of life, the majority of research has focused on pediatric cognitive outcome. Pre- and post-operative executive dysfunction and greater visuospatial than verbal intellectual deficits have been reported in children (Cavazzuti et al. 1983). Most research on adult patients suggests 
improved neuropsychological functioning following resection (Honeggar et al. 1998). While pre-treatment deficits have been observed in memory, verbal fluency, and executive skills (Donnet et al. 1999), postoperative improvements to normal functioning are common. Given frontal-subcortical circuitry involvement, patients are also at risk for psychiatric complications, specifically depression (Spence et al. 1995).

Cases of cognitive impairment in $\mathrm{CP}$ patients have been correlated with surgical approach and outcome. Despite the need for retraction of the frontal lobes with some approaches, frontal dysfunction remains relatively uncommon. Donnet et al. (1999) found post-operative impairment in 5 of $22 \mathrm{CP}$ patients on episodic memory and executive measures. However, poor surgical outcome (e.g., hematoma and edema), radiotherapy, and previous alcoholism were present in these 5 cases, confounding the results. There is one reported case of anterograde amnesia in a $\mathrm{CP}$ patient who sustained damage to the mammillary bodies (MB) alone (Tanaka et al. 1997). Following resection, the patient improved on immediate memory, but remained amnestic after delays. Executive functions and other skills were largely unimpaired and there was no retrograde amnesia.

Anterograde amnesia is typically associated with mesial temporal lobe damage, such as in the patient HM (Scoville \& Milner 1957), but has been described in association with other forms of pathology, such as lesions to the thalamus (Malamut et al. 1992), transection of the fornix and mammillothalamic tracts (Dusoir et al. 1990), and basal forebrain lesions (Abe et al. 1998). The capability of discrete MB lesions to produce amnesia is unclear. Certainly, atrophy of the mammillary bodies is a hallmark of Korsakoff syndrome (KS), which produces both retrograde and anterograde amnesia. Howev- er, portions of the thalamus and hippocampus are also involved in KS (Aggleton \& Brown 1999, Fama et al. 2004) and recent reports have shown both amnesia in the presence of intact MBs and damaged $\mathrm{MB}$ in alcoholic patients who did not suffer from amnesia (Sullivan et al. 1999). Animal studies suggest that selective MB lesions are not sufficient (Zola-Morgan et al. 1989), but as noted above, Tanaka and colleagues (1997) reported a case of persistent anterograde amnesia in a patient with atrophic MB and intact surrounding memory structures. Thus the role of the MB in amnesia remains imprecise.

Recently, Hildebrandt et al. (2001) presented a case of bilateral MB atrophy with an infarct of the left MB. They evaluated the model proposed by Aggleton and Brown (1999) in which a dissociation of free recall (impaired) and recognition (spared) is proposed for bilateral damage to structures within the Circuit of Papez, such as the MB. According to this model, recognition memory should be relatively spared because feelings of familiarity are hypothesized to be supported by perirhinal cortex. Their patient showed impaired anterograde (episodic) memory, poor source and recency memory, and relatively spared learning over repetition. Recognition results were mixed with spared recognition only when distractors were items not previously used (i.e., the patient was susceptible to interference). These results suggest limited support for the Aggleton \& Brown model, due to the fact that both free recall and recognition were impaired in this patient. Further, they suggested that the diencephalon is implicated in memory for context, a function typically associated with the frontal lobes and thalamus, but not previously with the MB (Dobbins et al. 2002, Smith \& Milner 1983).

Thus, the specific role of the MB in human memory remains in question, although there 
is accumulating evidence of its importance. To our knowledge, we present the first case of bilateral MB removal in the context of $\mathrm{CP}$ resection. We present the case of a 51-year old woman who suffered a disconnection of the hippocampal-limbic system through loss of the MB and columns of the fornix. This unique pathology allows for the exploration of memory functions including 1) recall vs. recognition, 2) learning, 3) implicit memory and priming and 4) contextual memory, as measured by a frequency judgment task (see below). We anticipated that recognition would be more preserved than recall, that new learning would be severely impaired and that implicit and contextual memory would be spared.

\section{Method}

\section{Case Report}

BR was a 51-year old, right-handed Caucasian woman with a GED education and no prior neurologic or psychiatric history (negative for prior depression), who presented to her physician with a visual field defect and was diagnosed with a CP by MRI 6 months later. Treatment was by surgical resection via right orbitofrontal craniotomy one month post-diagnosis. Surgical notes described a difficult resection due to adherence of the CP to surrounding structures and difficulty differentiating it from healthy tissue. Her hospital course was complicated by panhypopituitarism, lower extremity deep venous thromboses and pulmonary emboli. Throughout her hospital stay, the patient was described as disoriented, confused and agitated. She was referred for a neuropsychological evaluation approximately 10 weeks after surgery due to persistent memory deficits.

\section{Post-Operative Brain Imaging Findings}

MRI 8 weeks post surgically with diffusion-weighted images revealed the absence of the pituitary stalk, mammillary bodies, and columns of the fornix, a small (4 mm) ACoA aneurysm, and small hemorrhage in the right caudate head and anterior putamen. The aneurysm and hemorrhage were new findings not seen on pre-surgical imaging. CT 4 months post surgically showed decreased attenuation surrounding the anterior horn of the right lateral ventricle, suggestive of surgical trauma or encephalomalacia, and the previous lacune. Repeat MRI 9 months post surgically showed the same findings, with decreased size of the basal ganglia lacune and a small number of punctate hyperintensities in the deep white matter of the frontal and parietal lobes (Figure 1).

\section{Neuropsychological Test Results}

The patient completed a comprehensive neuropsychological examination 10 weeks post-surgically. She was grossly disoriented to day and date, stating that she lived in another state (she had moved 3 years previously) and miscalculating her age by one year. She was unaware of the surgery and had patchy retrograde amnesia for a 5-6 month period preceding the surgery (e.g. she was unable to recall Christmas or that her husband had retired). Anterograde amnesia was evident in her inability to store new memories for longer than 10 minutes, with only one "overnight" memory reported. She stated, "I don't know what I have done and what I have dreamed." Affect alternated between tearful and restricted, with lack of facial expressiveness and poor eye contact. Reported mood was depressed with associated significant functional decline. Spontaneous speech was slow. 
Sensorimotor testing revealed bilateral lower quadrant visual field defects (presumably the result of the $\mathrm{CP}$ and resection in proximity to the optic chiasm and tracts), bilateral motor slowing and diminished strength, dysdiadochokinesis, and slow acquisition of a new motor sequence. Although there was no obvious indication of a marked dysexecutive syndrome (e.g., difficulty with set shifting, inap- propriate behavior) and she had some awareness of her deficits, she displayed some evidence of apathy (reduced initiation) and perseveration (echopraxis on a go/no-go task).

Complete results of the formal neuropsychological exams are presented in Table I. Although no premorbid cognitive assessment was completed, her word knowledge (Vocab-

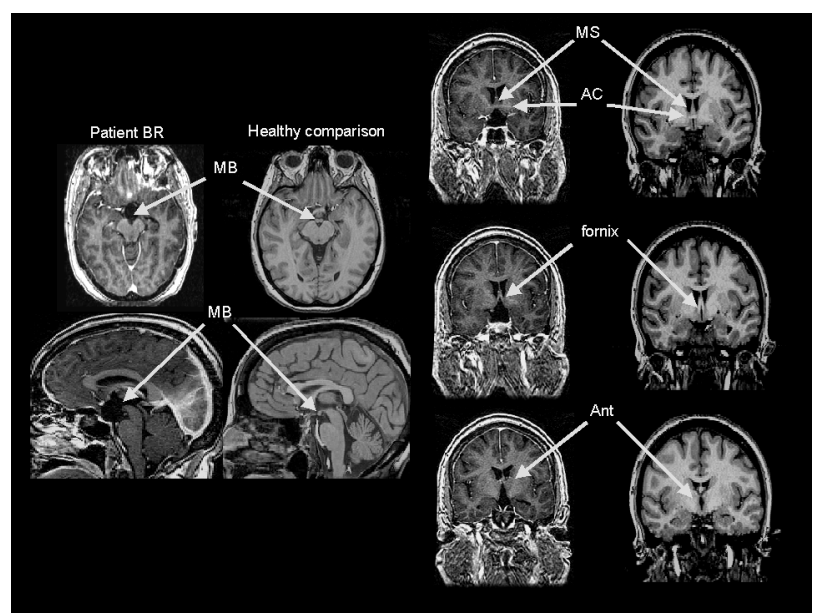

Figure 1. Shows axial, sagittal, and coronal images of pt. BR (left) and a healthy 44 y.o. female (right) for comparison (left side of image = left side of subject). The images clearly demonstrate the absence of the mamillary bodies in the patient, as well as the loss of the columns of the fornix inferiorly. In the coronal montage, the anterior commissure can be visualized. Immediately anterior to this structure is the relatively intact medial septal nucleus, suggesting that the precommissural fornix is intact as well. The absence of the mamillary bodies indicates that the mammillothalamic tract must also be lesioned. The remaining question of whether a few fibers remain that connect directly from the fornix to the anterior nuclear group can not be definitively resolved from these images. Also present, but not visible in these images is a small residual hemorrhage in right caudate head. $\mathrm{AC}=$ Anterior Commissure; $\mathrm{Ant}=$ Anterior nuclear group of thalamus; $\mathrm{MB}=$ Mammillary bodies; MS = Medial Septum; fornix = Post-commissural fornix.

ulary) score was average/low average, consistent with her education; this is often used as a measure of premorbid functioning because Vocabulary score correlates highly with full scale IQ (Wechsler 1997). Confrontation naming was above average and is correlated with education and intellect. To summarize the exam, performance was grossly normal (within 1.5 standard deviations of the mean) on most tests of visuomotor skills, language, simple attention, and executive functioning. In contrast, both visual and verbal memory were severely impaired, with flat learning curves, absent recall at delay, and no improvement with recognition procedures. Striking perseveration was seen across tasks. She was also impaired on tests of complex attention that required manipulation of information in working memory (Auditory Consonant Trigrams and Paced Auditory Serial Additions Test). She returned for follow-up at 30 weeks, at which time an abbreviated battery with repeated tests was given, in addition to several new memory tests. Some improvements in 
executive skills were demonstrated (Wisconsin Card Sorting Test and verbal fluency) with no significant change in attention or working memory. However, perseveration and lack of inhibition on motor testing remained and performance on two executive tests did not improve (Similarities and Trails B). No functional improvement in memory was achieved, with most recall scores at zero after a distraction. However, the patient had stored several memories for 24 hours and could report that she had undergone surgery, although one month of retrograde amnesia and day, date, and time disorientation remained. Depression and functional status improved, but remained abnormal (e.g., continued flat affect, psychomotor retardation and dependency).

To further explore her memory system, two experimental tasks, a word stem priming test (Haut et al. 1995) and a frequency memory measure (i.e., ability to estimate the number of times a word was read from a long list, without being cued to attend to this) were administered. On the word stem task, B.R. was shown 60 words printed on cards and was asked for each word to answer whether it either rhymed with another word (acoustic processing) or was a member of a category (semantic processing), thereby assuring some level of processing of each word. Following this presentation, she was asked to complete 3 letter word stems with the first word that came to mind. Half the stems were from the presentation list and half were new. She showed intact priming with a $33 \%$ benefit from prior exposure (normal $=40 \%$; Haut $e t$ al. 1995); recognition of which words were on the list remained at chance. Frequency memory was assessed by presenting a study list of words each between one and six times. B.R. was then asked to estimate how many times she had heard each word on the list. Her judgments were normal at $78 \%$ accuracy (controls $=76 \%+/-8 \%$; Haut et al. 2001), but she showed impaired recognition judgment when asked to identify the target words from among distractor words (67\% accuracy compared to $98 \%+/-1.3 \%$ in controls).

\section{Discussion}

We present a case of loss of the bilateral mammillary bodies, columns of the fornix, and mammillothalamic tracts in a patient who underwent a difficult surgical resection of a $\mathrm{CP}$. The case displays a rare and severe memory impairment with comorbid persistent depression. This unique lesion allowed us to explore the memory system and to address questions regarding the role of the $\mathrm{MB}$ in recall, recognition, learning, implicit memory and contextual memory. The presence of persistent depression may also be related to the subcortical damage sustained by this surgery; similar mood alterations are reported in stroke patients with left hemisphere lesions to the basal ganglia (Robinson \& Starkstein 1989).

Neuropsychological testing revealed normal range performance on tests without episodic memory demands at 30 weeks postoperatively; however, retrograde and anterograde amnesia were present. Importantly, we did not observe a dissociation between free recall and recognition, as proposed by the Aggleton \& Brown model (1999) in cases of bilateral damage to the Papez circuit. Overt recognition ranged from low average to severely impaired, consistent with the patient described by Hildebrandt (2001). Impaired consolidation is suggested by poor free recall with no benefit from recognition cues.

In contrast, BR showed spared priming and context memory. Priming is typically preserved in patients with amnesia and is mediated by a cortical network independent of the mesial temporal structures (e.g., Schachter 
Table I

Neuropsychological Test Scores at 10 and 30 Weeks Post CP Resection

\begin{tabular}{|c|c|c|c|c|}
\hline Test & $\begin{array}{l}10 \text { Weeks Post-Op } \\
\text { Raw Score }\end{array}$ & Percentile & $\begin{array}{l}30 \text { Weeks Post-Op) } \\
\text { Raw Score }\end{array}$ & Percentile \\
\hline MMSE & 22 & $<1$ & 21 & $<1$ \\
\hline \multicolumn{5}{|l|}{ Visuospatial } \\
\hline Digit Symbol & $\begin{array}{l}50 \text {, pairing }=0, \text { free } \\
\text { recall }=2\end{array}$ & $\begin{array}{l}9, \text { Pairing/ } \\
\text { recall = floor }\end{array}$ & $\begin{array}{l}\text { Pairing }=1, \\
\text { recall }=6\end{array}$ & $\begin{array}{l}\text { Pairing }=1, \\
\text { recall }=27\end{array}$ \\
\hline Judgment Line Orientation & 17 & 9 & & \\
\hline Block Design & 25 & 16 & & \\
\hline \multicolumn{5}{|l|}{ Span/Working Memory } \\
\hline Digit Span & $\begin{array}{l}13 \text { ( } 5 \text { forward, } \\
4 \text { back) }\end{array}$ & 16 & $\begin{array}{l}13 \text { ( } 6 \text { forward, } \\
3 \text { back) }\end{array}$ & 16 \\
\hline Auditory Consonant Trigrams & $32 / 60,27$ perseverations & & $30 / 60$ & \\
\hline Trail Making: A \& B & $46 \mathrm{sec}, 78 \mathrm{sec}$ & 21,37 & $28 \mathrm{sec}, 81 \mathrm{sec}$ & 79,32 \\
\hline Paced Auditory Serial Additions Test & Unable to complete & & Unable to complete & \\
\hline \multicolumn{5}{|l|}{ Language } \\
\hline Vocabulary & 37 & 25 & & \\
\hline Boston Naming Test & 57 & 63 & & \\
\hline COWAT/Animal & 22 (10 animals) & 8,8 & 33 (14 animals) & $\begin{array}{l}39,30 \\
\text { Memory }\end{array}$ \\
\hline Logical Memory I \& II & $\begin{array}{l}\mathrm{I}=8, \mathrm{II}=0, \\
\text { recognition }=13 / 20\end{array}$ & $\mathrm{I}=2 \mathrm{II}$ = floor & $\begin{array}{l}\mathrm{I}=16, \mathrm{II}=1, \\
\text { recognition }=11 / 20\end{array}$ & $\begin{array}{l}\mathrm{I}=16 \\
\mathrm{II}=\text { floor }\end{array}$ \\
\hline Hopkins VLT (Trials 1-3,4) & $5,3,6$, Delay $=0$ & $\begin{array}{l}<1 \text { (total) } \\
\text { Delay = floor }\end{array}$ & $\begin{array}{l}5,6,5, \\
\text { Delay }=0\end{array}$ & $\begin{array}{l}<1 \text { (total) } \\
\text { Delay = floor }\end{array}$ \\
\hline HVLT Recognition Discrimination & 10 & 16 & 8 & $<1$ \\
\hline Recognition Memory Test & $24 / 50$ words, $34 / 50$ faces & $<1,1$ & 31 words, 32 faces & 1,1 \\
\hline Visual Reproduction I \& II & $\mathrm{I}=20, \mathrm{II}=4, \operatorname{recog}=1 / 5$ & $\mathrm{I}=6 \mathrm{II}$ = floor & $\begin{array}{l}\mathrm{I}=23, \mathrm{II}=0, \\
\operatorname{recog}=2 / 5\end{array}$ & $\begin{array}{l}\mathrm{I}=13 \\
\mathrm{II}=\text { floor }\end{array}$ \\
\hline \multicolumn{5}{|l|}{ Executive } \\
\hline Stroop Color and Word & $90,70,34$ & $19,25,14$ & & \\
\hline Similarities & 17 & 16 & 16 & 16 \\
\hline Wisconsin Card Sorting Test & $\begin{array}{l}42 \text { errors, } \\
32 \text { perseverations }\end{array}$ & $14-27$ & $\begin{array}{l}20 \text { errors, } \\
3 \text { perseverations }\end{array}$ & $39-58$ \\
\hline
\end{tabular}

Note: percentile scores below 7 are equivalent to 1.5 standard deviations below the mean and generally represent impairment.

1987, Squire \& Graf 1987, Goshen-Gottstein et al. 2000). Our patient showed intact contextual memory (e.g. frequency memory, source memory), which has previously been shown to be preserved in patients with MTL damage and is thought to depend on frontoparietal networks that mediate working memory and metamemory (e.g., Haut et al. 2001, Smith \& Milner 1988, Jurado et al. 1997 ). Using the same frequency memory task used with BR, Haut et al. (2001) found fMRI activation during the frequency memory task in healthy controls in the ventrolateral prefrontal cortex. They further showed a dissociation between frontal and hippocampal activation with frequency versus recognition judgments (no hippocampal activation for frequency), supporting the role of the frontal lobes in memory for frequency of occurrence. This finding contrasts with the patient described by Hildebrandt (2001) and contradicts their proposal of MB involvement in this form of contextual memory. Instead, the previous literature implicating a network of cortical structures (prefrontal cortex, posterior parietal cortex, posterior cingulate, and pre- 
cuneus) for contextual memory is supported. Importantly, modification of recognition memory through practice has been more promising than of frequency memory (Zacks et al. 1982). This may have implications for interventions with patients such as BR, and may offer an explanation for her perceived improvements in "memory" and functional status.

We propose that the amnesia in this patient, whose mesial temporal structures are intact, is due to disruption of the white matter pathways responsible for transmission of information through the limbic system. This finding is similar to previous cases (e.g., Dusoir et al. 1990, Abe et al. 1998, Tucker et al. 1988), in which global amnesia resulted from disconnection of the hippocampus from the MB and basal forebrain. The presence of significant temporal disorientation, which has been associated with the anterior nuclei (Clarke et al. 1994), and other thalamic and fornix lesions (Shuren et al. 1997, Yasuno et al. 1999) further supports our contention of a functional disconnection of the hippocampus and thalamus.

Brain imaging on BR clearly demonstrates the loss of the MB and the columns of the fornix, but it is unclear whether traces of the postcommissural fornices may have been spared. These fibers synapse directly with the anterior nuclei of the thalamus and may support memory function in the absence of the MB (Carpenter \& Sutin 1983). Connections to the thalamus via the amygdalofugal pathway are another potential route from the limbic structures to the thalamus (Crosson 1992). BR's ability to recall a few bits of information by 30 weeks post-surgery may be accounted for by the presence of these pathways. Further advances in imaging white matter pathways (i.e., diffusion tensor imaging) may provide the detail necessary to answer these questions in the future.
In summary, this case illustrates three important points: 1.) given the typical location of the tumor, CP resection can present significant (albeit rare) neuropsychiatric risks to the patient. 2.) amnesia can occur in the presence of intact hippocampi, due to disruption of their white matter connections to the diencephalon. 3.) this case provides new support for a distributed cortical network, but not mammillary body, involvement in contextual memory.

\section{Acknowledgments}

We thank B.R. and her family for graciously volunteering their time and effort. We also thank David Kareken, Ph.D. for his helpful comments on earlier versions of this paper, Gary Marano, M.D. for assistance with neuroradiologic acquisition and interpretation, and Lyn Harper Mozley, Ph.D. for manuscript suggestions.

\section{References}

Abe K, Inokawa M, Kashiwagi A, Yanagihara T. Amnesia after a discrete basal forebrain lesion. J Neurol Neurosurg Psychiatry 1998; 65: 126-30.

Aggleton JP, Brown MW. Episodic memory, amnesia, and the hippocampal-anterior thalamic axis. Behav Brain Sci. 1999; 22: 425-44.

Cansino S, Maquet P, Dolan RJ, Rugg MD. Brain activity underlying encoding and retrieval of source memory. Cereb.Cortex 2002; 12: 1048-56.

Carpenter MB, Sutin J. Human Neuroanatomy ( $8^{\text {th ed.) }}$. Baltimore: Williams and Wilkins; 1983

Cavazzuti V, Fischer E, Welch K, Belli J, Winston K. Neurological and psychophysiological sequelae following different treatments of craniopharyngioma in children. $J$ Neurosurg 1983; 59: 409-417.

Clarke S, Assal G, Bogousslavsky J, Regli F, Townsend DW, Leenders KL, et al. Pure amnesia after unilateral left polar thalamic infarct: topographic and sequential neuropsy- 
chological and metabolic (PET) correlations. J Neurol Neurosurg Psychiatry 1994; 57: 27-34.

Crosson, B. Subcortical Functions in Language and Memory. 1. New York: Guilford Press; 1992.

Dobbins I, Foley H, Schacter D, Wagner A. Executive control during episodoc retrieval: Multiple prefrontal processes subserve source memory. Neuron 2002; 35: 989996.

Donnet A, Schmitt A, Dufour H, Grisoli F. Neuropsychological follow-up of twenty two adult patients after surgery for craniopharyngioma. Acta Neurochir 1999; 141: 10491054 .

Dusoir H, Kapur N, Byrnes DP, McKinstry S, Hoare RD. The role of diencephalic pathology in human memory disorder. Evidence from a penetrating paranasal brain injury. Brain 1990; 113 ( Pt 6): 1695-1706.

Fama R, Marsh L, Sullivan E. Dissociation of remote and anterograde memory impairment and neural correlates in alcoholic Korsakoff syndrome. J Intnl Neuropsychol Soc 2004; 10; 427-441.

Goshen-Gottstein Y, Moscovitch M, Melo B. Intact implicit memory for newly formed verbal associations in amnesic patients following single study trials. Neuropsychology 2000; 14: 570-578.

Haut MW, Arias R, Moran M, Leach S, Parsons MW, Kuwabara KH. Neural activation during frequency-memory performance. Neuropsychology 2001; 15: 568-575.

Haut M, Young J, Cutlip W, Callahan T, Haut A. A case of bilateral thalamic lesions with anterograde amnesia and impaired implicit memory. Arch Clin Neuropsychol 1995; 10: 555-566.

Hildebrandt H, MS, B-MB, Goebel S, Eilers N. Are some memory deficits unique to lesions of the mammillary bodies? J Clin Exp Neuropsychol 2001; 23: 490-501.

Honeggar J, Barocka A, Sadri B, Fahlbusch R. Neuropsychological results of craniopharyngioma surgery in adults: A prospective study. Surg Neurol 1998; 50: 19-29.

Jurado M, Junque C, Pujol J, O’Beal. Impaired estimation of word occurrence frequency in frontal lobe patients. Nueropsychologia 1997; 35: 635-641.

Malamut BL, Graff-Radford N, Chawluk J, Grossman RI, Gur RC. Memory in a case of bilateral thalamic infarction. Neur. 1992; 42: 163-169.

Robinson R G, Starkstein S E. Mood disorders following stroke: new findings and future directions. J Geriatr Psychiatry 1989; 22(1): 1-15.

Schacter DL. Implicit expressions of memory in organic amnesia: learning of new facts and associations. Hum Neurobiol 1987; 6: 107-118.
Scoville W, Milner B. Loss of recent memory after bilateral hippocampal lesions. JNeurol Neurosurg Psychiatry 1957; 20: 11-12.

Shuren JE, Jacobs DH, Heilman KM. Diencephalic temporal order amnesia. J Neurol Neurosurg Psychiatry 1997; 62: 163-168.

Smith ML, Milner B. Estimation of frequency of occurrence of abstract designs after frontal or temporal lobectomy. Neuropsychologia 1988; 26: 297-306.

Smith ML, Milner B. Effects of focal brain lesions on sensitivity to frequency of occurrence [abstract]. Soc Neurosci Abstr 1983; 9: 30.

Spence SA, Taylor DG, Hirsch SR. Depressive disorder due to craniopharyngioma. J R Soc Med, 1995; 88: 637-638.

Squire L, Graf P. Strength and duration of priming effects in normal subjects and amnestic patients. Neuropsychologia 1987; 25: 597-604.

Sullivan EV, Lane B, Rosenbloom MJ, Deshmukh A, Desmond J, Lim KO et al. In vivo mammillary body volume deficits in amnestic and nonamnestic alcoholics. Alcohol Clin Exp Res 1999; 23: 1629-1636.

Tanaka Y, Miyazawa Y, Akaoka F, Yamada T. Amnesia following damage to the mammillary bodies. Neurology 1997; 48: 160-165.

Tucker DM, Roeltgen DP, Tully R, Hartmann J, Boxell C. Memory dysfunction following unilateral transection of the fornix: a hippocampal disconnection syndrome. Cortex 1988; 24: 465-472.

Yasuno F, Hirata M, Takimoto H, Taniguchi M, Nakagawa Y, Ikejiri Y, et al. Retrograde temporal order amnesia resulting from damage to the fornix. J Neurol Neurosurg Psychiatry 1999; 67: 102-125.

Zacks RT, Hasher L, Sanft H. Automatic encoding of event frequency: Further findings. J Exp Psychol Learn Mem Cogn 1982; 8: 106-116.

Zola-Morgan S, Squire LR, Amaral DG. Lesions of the hippocampal formation but not lesions of the fornix or the mammillary nuclei produce long-lasting memory impairment in monkeys. J Neurosci 1989; 9: 898-913.

Address of pcorrespondence:

Leigh Beglinger, Ph.D.

Department of Psychiatry

University of Iowa College of Medicine

200 Hawkins Dr., MEB 1-325

Iowa City, IA 52242

Phone: (319) 335-8765

Fax: (319) 353-3003

Email: leigh-beglinger@uiowa.edu

USA 